\title{
Structure, Stability, and Hydration of a Polypeptide in AOT Reverse Micelles
}

\author{
Stéphane Abel, ${ }^{\dagger} \S$ Marcel Waks, ${ }^{\dagger}$ Wladimir Urbach, ${ }^{\ddagger}$ and Massimo Marchi ${ }^{\star}, \S$ \\ Commissariat à l'Energie Atomique, DSV-DBJC-SBFM, Centre d'Etudes, Saclay, 91191 Gif-sur-Yvette Cedex, \\ France, Laboratoire d'Imagerie Paramétrique, CNRS UMR7623, F-75006 Paris Cedex 06, France, \\ Laboratoire de Physique Statistique, CNRS UMR8550, Ecole Normale Supérieure, 24 Rue Lhomond, \\ F-75231 Paris Cedex 05, France
}

Received May 10, 2005; E-mail: mmarchi@cea.fr

It has been known for a time, both from experiments ${ }^{1}$ and theory, ${ }^{2}$ that confining a peptide chain within a geometrically restricted environment increases the relative stability of the folded state against unfolded states. This finding is of biological relevance as (in vitro) intrinsically disordered proteins have shown to be structured or folded in their native cellular environment where excluded volume effects are important. A convenient means to confine a protein within a certain region of space is through encapsulation in reverse micelles $^{3}$ (RM). In this communication, we provide theoretical evidence that the folded structure of a simple peptide, alanine zwitterionic octapeptide, or $\mathrm{A}_{8}$, unstable in solution, becomes stable in an RM of appropriate size. Our molecular dynamics simulations were carried out for realistic models of sodium 2-ethylhexylsulfosuccinate (AOT) RM in isooctane, simulated for an extended period of time. For the RM of the smaller size, we find that a helical structure is stable for the whole length of the simulation. On the contrary, the peptide very quickly takes an extended structure in larger micelles.

In a previous study, we developed a realistic molecular model for AOT reverse micelles in isooctane and reported on the structural properties of the micelles and their water core ${ }^{4}$ as a function of the water-to-surfactant molar ratio $\left(\mathrm{W}_{\mathrm{o}}=\left[\mathrm{H}_{2} \mathrm{O}\right] /[\mathrm{AOT}]\right)$. Here, we focus on the quaternary system $\mathrm{A}_{8} / \mathrm{AOT} /$ water/isooctane. Studies using both experiments ${ }^{5}$ and simulations ${ }^{6}$ have shown that in water at room temperature an octaalanine is unstable in its canonical $\alpha$-helix conformation. According to MD investigations, ${ }^{6}$ the peptide prepared as an $\alpha$-helix evolves to extended structures within a few hundred or thousand picoseconds of simulation, depending on the force field. This behavior makes octaalanine an ideally simple but physically relevant system to study confinement effects for protein secondary structure.

In this work, ${ }^{7}$ all simulations were performed in an isobaricisothermal (NPT) ensemble at $T=300 \mathrm{~K}$ and $P=0.1 \mathrm{MPa}$, as described in ref 8 . In a first series of MD runs (see ref 4 ), two reverse micelles with 64 and 82 molecules of AOT and 320 and 574 molecules of water (named RM64 and RM82 and corresponding to $\mathrm{W}_{\mathrm{o}}=5$ and 7 , respectively) were simulated. For the current study, an additional series of runs, for a total of $26 \mathrm{~ns}$ (for both systems we ran two simulations of 3 and $10 \mathrm{~ns}),{ }^{7}$ was performed by inserting a canonical $\alpha$-helical octaalanine in the center of the RM64 and RM82 water core. In doing so, water molecules in contact with the peptide were removed, thus leaving 306 and 557 waters in the hydrophilic cores of RM64 and RM82, respectively. The new systems, named $\mathrm{RM} 64+\mathrm{A}_{8}$ and $\mathrm{RM} 82+\mathrm{A}_{8}$, had waterto-surfactant molar ratios of $\mathrm{W}_{\mathrm{o}}=4.78$ and 6.79 , respectively. The small reduction of the number of water molecules (less than 5 and $3 \%$ between identical micelles) allows a direct comparison with

\footnotetext{
$\dagger$ Laboratoire d'Imagerie Paramétrique.

₹aboratoire de Physique Statistique.

$\S$ Commissariat à l'energie Atomique.
}

the peptide-free RM. For all AOT RMs studied here, the solvent concentration was chosen to be compatible with the $\mathrm{L}_{2}$ phase of AOT/water/isooctane. ${ }^{9}$

We first notice ${ }^{10}$ that RMs with and without the peptide are of ellipsoidal shape. As discussed in ref 4, there is experimental evidence that AOT RMs are nonspherical (see, for instance, ref 11). The ratio between the major and the minor semi-axis, $\langle a / c\rangle$, for each micelle is within $1.66\left(\mathrm{RM} 64+\mathrm{A}_{8}\right)$ and $1.77\left(\mathrm{RM} 82+\mathrm{A}_{8}\right)$ units. Inserting $A_{8}$ modifies this semi-axis ratio for both micelles and their water cores. For RM64, $\langle\mathrm{a} / \mathrm{c}\rangle$ increases, whereas it decreases for RM82. The radius of gyration $R_{\mathrm{g}}$, an estimate of the $\mathrm{RM}$ dimensions, is less affected by the peptide as it changes only a few percent. The largest variation is seen for $\mathrm{RM} 82 \rightarrow \mathrm{RM} 82+\mathrm{A}_{8}$, where $R_{\mathrm{g}}$ of the water core goes from 14.6 to $16.6 \AA$. This is probably related to the structural modifications of the confined peptide (see below).

The radial density profiles computed for all components of our systems also show that for the peptide-containing RMs the AOT aliphatic chains protect the water core from the oil and that a large interfacial zone exists occupied by the AOT headgroups and $\mathrm{Na}^{+}$ ions. We also observe that octaalanine is well-confined within $\mathrm{RM} 64+\mathrm{A}_{8}$ and $\mathrm{RM} 82+\mathrm{A}_{8}$ micelles, since no surface contacts ${ }^{12}$ between the peptide and the solvent are detected during the simulation time. Calculations of the pair density correlation functions, $\rho(\mathrm{r})$, show that insertion of the peptide does not modify the hydration of the AOT headgroups. Indeed, we find that the number of water molecules bound to the AOT sulfonate groups remains very close to that found in peptide-free micelles with changes of only 0.4 units (see Table 1 in the Supporting Information). We also notice that $\mathrm{A}_{8}$ produces small changes on the residence times, $\tau_{\mathrm{w}}$, of the waters bound to the AOT headgroups of RM82, whereas its effect is larger for RM64. If $\tau_{\mathrm{w}}$ and $\tau_{\mathrm{w}}$ bulk are the water residence times of the system under consideration and of the bulk, the retardation ratio is defined as $\tau_{\mathrm{w}} / \tau_{\mathrm{w}}$ bulk. We find that the retardation of 2.7 in RM82 changes very little, to 3.3, in $\mathrm{RM} 82+\mathrm{A}_{8}$. On the contrary, the retardation of 3.1 for RM64 is almost doubled, to 5.5 , by the peptide. This means that water is slowed considerably in RM64+ $\mathrm{A}_{8}$. This likely prevents the peptide to break its intramolecular $\mathrm{H}$-bonds and might have crucial implications for the kinetic stability of the peptide folded structure.

In Figure 1, we show typical conformations of A8 in the RM64 and RM82 micelles. We first notice that the peptide $\alpha$-helical secondary structure is conserved for RM64+A8, whereas for $\mathrm{RM} 82+\mathrm{A} 8$ the intramolecular hydrogen bonds (IHB) are disrupted. To quantify this behavior, we counted the IHB between the carbonyl and the amide groups separated by three residues, using geometrical and energy criterions ${ }^{13}$ (see Supporting Information). In bulk water, no IHB persisted after only a few tens of picoseconds, confirming previous results in ref 6 . Conversely, in the RM64+A8 simulation 


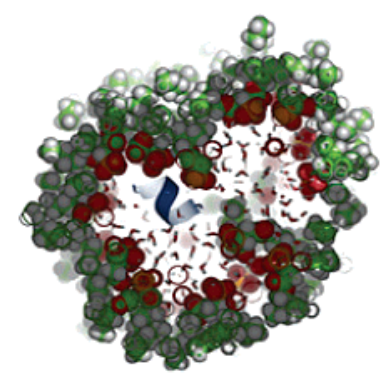

(a)

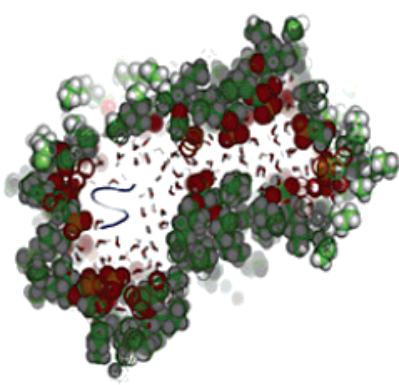

(b)
Figure 1. Views of the reverse micelles $\mathrm{RM} 64+\mathrm{A}_{8}$ (a) and $\mathrm{RM} 82+\mathrm{A}_{8}$ (b) at the end of the NPT simulations. These are views of the simulation box clipped by an orthogonal plane to the $X$-axis. The peptide is drawn as a cartoon in blue color. The atoms of AOT are represented by spheres, whereas the water atoms are represented by sticks. For visual clarity, the $\mathrm{Na}^{+}$ions and the isooctane molecules are not shown. The pictures were drawn with PyMOL. ${ }^{14}$

three out of four IHBs were maintained during the full length of the simulation, with some fast, less than 300-ps long, hydrogen bond exchanges with water. In RM82+A8, during the 3-ns run two of the bonds were lost after $10 \mathrm{ps,} \mathrm{whereas} \mathrm{the} \mathrm{remaining} \mathrm{two,}$ $\mathrm{CO}(1) \rightarrow \mathrm{NH}(5)$ and $\mathrm{CO}(2) \rightarrow \mathrm{NH}(6)$, lasted for 0.5 and $1.5 \mathrm{~ns}$, respectively. In the 10-ns run, the IHB kinetic was slower and only the $\mathrm{CO}(2) \rightarrow \mathrm{NH}(6)$ IHB persisted continuously for the entire trajectory. At the same time, deviations from the initial $\alpha$-helical structure remained below $1.8 \AA$ for both RM64+A8 simulations but reached as high as $4.2 \AA$ for the shorter and $3 \AA$ for the longer $\mathrm{RM} 82+\mathrm{A} 8$ simulation.

We find that a possible explanation for the stability of the $\mathrm{A}_{8}$ $\alpha$-helix in RM64 resides in the competition for hydration water between the AOT headgroup and the peptide. In the RM64 system, the quantity of water not bound to the sulfonate group is very limited, preventing the full hydration of $\mathrm{A}_{8}$. This was confirmed by two extra simulations we carried out on $\mathrm{A}_{8}$ in the modified environments: (1) a RM64 system where the sulfonate group in AOT and the $\mathrm{Na}^{+}$ions were removed, for a system electrically neutral, designated as RM64+ $\mathrm{A}_{8} *$ and (2) A droplet of 306 water molecules, the same number as in RM64, in isooctane, named DROP. The two new systems were run in the same conditions of temperature and pressure as the other systems for 3 and $2.5 \mathrm{~ns}$, respectively. In both cases, we observe the unfolding of the peptide and then its migration to the interface between water and the modified detergent/oil phases. In the case of RM64+ $\mathrm{A}_{8} *$, the waters represent about $9 \%$ of the total surface of the solute in direct contact with the oil, and 20 modified detergent molecules are found in the solvent region at the end of the simulation run. The peptide needs $0.5 \mathrm{~ns}$ to unfold to an extended structure and $1.8 \mathrm{~ns}$ to get in stable contact with solvent and detergent molecules. For the DROP system, the unfolding to an extended structure is faster, $0.2 \mathrm{~ns}$, and its migration to the oil/water interface occurs in $0.25 \mathrm{~ns}$.

This communication has shown that the major force affecting the stability of octaalanine in small size RM of AOT is hydration. In our simulations, the detergent and the peptide polar groups have competing affinities with water: Hydration is stronger for the AOT sulfonate groups and the $\mathrm{Na}^{+}$ions than for $\mathrm{A}_{8}$. The properties of micellar water are known experimentally to differ significantly from those of the bulk ${ }^{15}$ and affect the confined peptides/proteins structures. ${ }^{3}$ In our simulations, we find that in the smallest RM, the dynamic of water is considerably slowed with respect to the bulk, 5.5 times, whereas not enough water is available to solvate the carbonyl and amide groups of the peptide. To conclude, our results provide a strong evidence of the crucial role played by hydration on the structure of peptides in confined hydrophilic environments. Hence, both hydration and excluded volume effects ${ }^{2}$ need to be taken into account to study the secondary structure stability of proteins in restricted spaces such as RM, nanoporous silica, and the drug nanocarriers as well as the interior of ribosomes, or regions of steric occlusion inside the cell.

Acknowledgment. S.A. is grateful for financial support from Association de gestion du fonds pour l' insertion professionnelle des personnes handicapées (AGEFIPH). Our simulations were in part carried out at the CEA high performance computing facilities in Bruyères Le Chatel.

Supporting Information Available: The details of our simulation protocols and an extended analysis of our results are reported in the Supporting Information. This material is available free of charge via the Internet at http://pubs.acs.org.

\section{References}

(1) (a) Thompson, K. F.; Gierash, L. M. J. Am. Chem. Soc. 1984, 12, $3648-$ 3652. (b) Gallay, J.; Vincent, M.; Nicot, C.: Waks, M. Biochemistry 1987 26, 5738-5747. (c) Eggers, D. K.; Valentine, J. S. Protein Sci. 2001, 10 250-261. (d) Bolis, D.; Politou, A. S.; Kelly, G.; Pastore, A.; Temussi, P. A. J. Mol. Biol. 2004, 336, 203-212. (e) Eggers, D. K.; Valentine, J. S. Protein Sci. 2001, 10, 250-261.

(2) (a) Minton, A. P. Biophys. J. 1992, 63, 1090-1100. (b) Zhou, H. X Dill, K. A. Biochemistry 2001, 40, 11289-11293.

(3) (a) Luisi, P. L.; Magid, L. J. Crit. Rev. Biochim. 1986, 20, 409-74. (b) Nicot, C.; Waks, M. Biotechnol. Genet. Eng. Rev. 1996, 13, 267-314. (c) Shastry, M. C. R.; Eftink, M. R. Biochemistry 1996, 35, 4094-4101. (d) Thena de Gomez Puyou, M.; Gomez-Puyou, A. Crit. Rev. Biochem. Mol. Biol. 1998, 33, 53-89. (e) Fiori, S.; Renner, C.; Cramer, J.; Pegorano, S.; Moroder, L. J. Mol. Biol. 1999, 291, 163-175. (f) Lundgren, J. S. Heitz, M. P.; Bright, F. V. Anal. Chem. 2000, 67, 3775. (g) Peterson, R. W.; Anbalagan, K.; Tommos, C.; Wand, A. J. J. Am. Chem. Soc. 2004, 126, 9498-9499.

(4) Abel, S.; Sterpone, F.; Bandyopadhyay, S.; Marchi, M. J. Phys. Chem. B 2004, 108, 19458-19466.

(5) Chakrabartty, A.; Baldwin, R. L. Adv. Protein Chem. 1995, 46, 141150.

(6) (a) Weber, W.; Hunenberger, P. H.; McCammon, J. C. J. Phys. Chem. B 2000, 104, 3668-3675. (b) Margulis, C. J.; Stern, H. A.; Berne, B. J. J. Phys. Chem. B 2002, 106, 10748-10752.

(7) See the Supporting Information for a full description of the simulation parameters and procedures. For each RM containing peptide, we ran two simulations with different initial conditions of 3 and $10 \mathrm{~ns}$, respectively. If not specified, results reported in this communication are from the 10 ns trajectory.

(8) Marchi, M.; Procacci, P. J. Phys Chem. B 1998, 109, 5194-5102.

(9) Structure and reactivity in reverse micelles; Pileni, M. P., Ed.; Elsevier: Amsterdam, 1989.

(10) The structural results discussed below are summarized in Table 1 of the Supporting Information.

(11) Yano, J.; Milhofer, H. F.; Wachtel, E.; Garti, N. Langmuir 2000, 16, 999610004

(12) We compute the contact surface between species A and B as the sum of the surface of the Voronoi ${ }^{12 a}$ facets shared between all the atoms of $\mathrm{A}$ with those of B. For a reference to the Voronoi polyhedra the reader should refer to: (a) Voronoi, G. F. J. Reine Angew. Math. 1908, 134, 198-287. Our calculations were carried out according to ref $12 \mathrm{~b}$ : (b) Procacci, P.; Scateni, R. Int. J. Quantum Chem. 1992, 42, 1515-1528.

(13) An IHB was assumed to be formed if the distance between the donor $(\mathrm{NH})$ and acceptor $(\mathrm{CO})$ group was smaller than $0.25 \mathrm{~nm}$. For the energy criterion, we used the Kabsch and Sander function: An HB between $(\mathrm{NH})$ and $(\mathrm{CO})$ exists if the energy is $\leq-0.5 \mathrm{kcal} / \mathrm{mol}$ (for further details see Kabsch, W.; Sander, C. Biopolymers 1983, 22, 2577-2637).

(14) DeLano, W. L. The PyMOL Molecular Graphics System; DeLano Scientific: San Carlos, CA, 2002; http://pymol.sourceforge.net/.

(15) Levinger, N. Science 2002, 298, 1722-1723.

\section{JA053043U}

